\title{
User-Aware Cell Switch-Off Algorithms
}

\author{
Ibrahim Aydin*, Halim Yanikomeroglu ${ }^{\dagger}$, and Ümit Aygölü* \\ * Dept. of Electronics and Communication Engineering \\ Istanbul Technical University, 34469, Maslak, Istanbul, Turkey \\ $\dagger$ Dept. of Systems and Computer Engineering \\ Carleton University, Ottawa, Canada \\ Email: \{iaydin, aygolu\}@itu.edu.tr, halim@sce.carleton.ca
}

\begin{abstract}
In recent years, energy efficiency ("greenness") has become an important research topic in wireless networks. Environmental awareness and the increased cost of energy stimulate the research on this subject. In cellular networks, most of the power consumption takes place at the base stations (BSs). It is worth noting that the number of BSs has been steadily increasing since the 1G networks; moreover, this increase is expected to become even steeper in the foreseeable future with the advent of the small cell concept in the envisioned $5 G$ networks. It is also worth noting that in cellular networks the traffic demand (load) in space and time is getting increasingly heterogeneous. As a result, parts of network will likely be lightly loaded at certain times. In such situations, it only makes sense to switch off as many BSs as possible without jeopardizing the key performance indicators.

Although there in an increasing volume of literature on the cell switch-off (CSO) concept, to the best of our knowledge, there is no study which considers the user terminal (UT) power consumption as a key performance indicator, while the UT power efficiency indeed constitutes one of most important performance criterion in a mobile network. In many cases, switching off BSs for downlink energy efficiency may result in an uplink energy inefficiency, due to the fact that the UTs served by the switched off BSs will need to be connected to further away BSs. In this paper, we propose a heuristic CSO algorithm to achieve energy efficiency in the whole cellular network while taking into account the power consumption of UTs. We call the proposed algorithm as the useraware CSO algorithm.
\end{abstract}

Index Terms-green communications, cell switch-off, useraware cellular networks.

\section{INTRODUCTION}

Since 1980s, the number of subscribers and traffic volume in cellular networks have grown exponentially. At the same time the energy consumption in cellular networks have also increased substantially [1].

BSs are the main energy consumers in a wireless cellular network where typical consumption ranges between $0.5 \mathrm{~kW}$ and $2 \mathrm{~kW}$ [2], including all parts that consume energy. In order to be more specific, $50 \%$ to $80 \%$ of the energy consumption in cellular networks takes place in the base stations (BSs) [3]. The power consumption of a BS can be categorized in two parts: (a) Transmission power, (b) the power consumption of some internal equipment, such as the cooling system and antenna, which are independent from the transmission power. While the transmission power is related to the traffic load, the second part is constant and independent from the traffic load. Moreover, the second part causes the major power consumption of a BS; a BS consumes at zero load about $60-80 \%$ of the energy consumption at full load [4].

Another very important point about the traffic behaviour in a cellular network is that there is a significant imbalance of the BS traffic loads, i.e., $10 \%$ of the BSs carry about $50-60 \%$ of the aggregate traffic load [5]. So, a great majority of the BSs carry light traffic loads and can be managed to increase the energy efficiency. In addition to that, BSs in a cellular network are deployed in order to satisfy the maximum requested capacity at the peak hours. While the traffic is under the capacity most of a day, BSs are generally underutilized which causes significant waste of energy. Because of the reasons mentioned above, the idea of switching off some BSs according to their traffic load appears to be a promising way intuitively.

There are two main approaches in the cell switch-off (CSO) concept: a) Deterministic approach where the CSO is performed according to the instantaneous traffic information, b) statistical approach where the statistical behavior of traffic is used to execute the CSO algorithms. References [6], [7] and [8] are samples of the deterministic CSO approach. Reference [6] is one of the first papers on CSO where a deterministic model called "cell-zooming" is proposed. The cell-zooming algorithm adjusts the cell sizes according to their traffic conditions. This technique results in energy saving in cellular networks. When a cell has a light traffic load, the BS of this cell can work in sleep mode, while the neighboring BSs zoom out to cover the area of the sleeping BS. The cellzooming algorithm is improved in [7] where the improved version does not terminate at the point that the standard version terminates, rather the algorithm continues to check all the BSs to determine whether they can be switched off. It is shown that this simple modification yields a significant improvement in the performance.

In [9] and [10], the user association problem is modelled in a different way, where the formulation considers the QoS as well as energy saving of the whole network. A weight matrix is proposed to trade off the two aspects, QoS and traffic load; the association between UTs and BSs are performed according to this weight matrix. Reference [11] proposes a novel distributed CSO algorithm which is implemented by exchanging load related information messages among the cells iteratively. All 
the cells are divided into groups, and the cells, to be switched off, are determined in their group in a distributed manner by exploiting the traffic load imbalances of the group.

Reference [5] states that the aggregate network load can be defined as periodic and exhibit significant temporal correlation, but the individual BSs do not exhibit such properties. Therefore the statistical behavior of the aggregate traffic load can be exploited to make decisions about CSO. References [2], [12] and [13], develop analytical frameworks to find the optimum CSO techniques as a function of the daily traffic pattern. Reference [14] proposes a multi-objective framework for CSO in cellular networks. The optimization procedure is completely based on statistical information such as the average traffic load and the spatial traffic distribution.

The major concern of the aforementioned literature is to decrease the power consumption of the whole network and they do not deal with the effect of their CSO methods on UTs' power consumption. In this paper, we propose a heuristic CSO algorithm to achieve energy efficiency in the whole cellular network while considering the power consumption of UTs. When we implement the algorithm, the total network power consumption decreases without violating the QoS constraint, while the UTs' power consumption is not severely affected from the CSO process. As a result, we obtain cost reduction due to energy efficiency of the network and the increased battery lives of the UTs when we compare the proposed algorithm with other CSO algorithms.

The paper is organized as follows: Section II deals with the system model, the power control algorithm and the proposed user-aware CSO algorithm. The simulation results are given in Section III, and concluding remarks are made in Section IV.

\section{System Model and Problem Formulation}

A homogeneous network is assumed where all cells have the same structure and the same area as shown in Fig. 1. The UTs are uniformly distributed to whole area and the traffic demand of each UT is identical as given in Table I. The full buffer traffic model is assumed that all the UTs in the network always receive and transmit data. Deterministic approach is adopted that we take a snapshot of the network at a certain time to determine which BSs will be closed. The effect of instantaneous interference is not considered; instead the average interference is used.

The power consumption of a BS can be modeled as [15]

$$
P=\alpha P_{t x}+\beta,
$$

where $\alpha$ and $\beta$ are coefficients for BS and $P_{t x}$ is the transmission power of BS. In a typical case, the constant power $\beta$ dominates the total power consumption [15]. All the UTs have the same rate requirement which should be fulfilled by the network, otherwise the UT is assumed to be in the outage state. So the requested downlink rate of a UT can be given as

$$
R_{d l}=B_{m, i}^{D} \log \left(1+\frac{P_{m, i}^{D} / \mathrm{PL}\left(d_{m, i}\right)}{I_{i}^{D}+N_{0} N_{f} B_{m, i}^{D}}\right),
$$

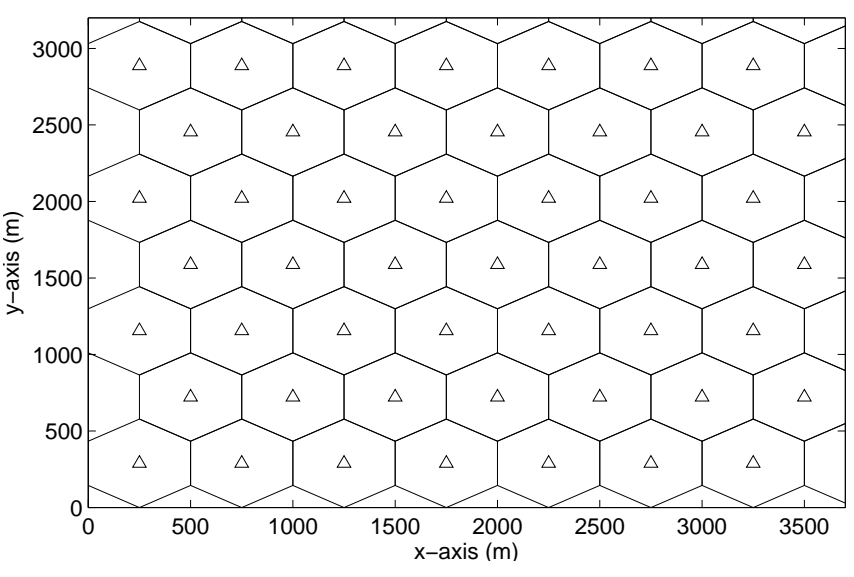

Fig. 1: Example of the network layout.

TABLE I: Definitions of some variables

\begin{tabular}{|l|l|}
\hline$R_{d l}$ & Fixed data rate for downlink (DL) \\
\hline$R_{u l}$ & Fixed data rate for uplink (UL) \\
\hline$A$ & Set of active BSs \\
\hline$S_{m}$ & Set of UTs associated with BS $m$ \\
\hline$P_{B S}$ & Maximum transmission power of a BS \\
\hline$B_{B S}$ & Total bandwidth for a BS \\
\hline$N$ & Number of all UTs \\
\hline$M$ & Number of BSs \\
\hline$N_{A}$ & Number of active BSs \\
\hline$B_{m, i}^{D}$ & Allocated downlink bandwidth of BS $m$ for UT $i$ \\
\hline$B_{m, i}^{U}$ & Allocated uplink bandwidth of BS $m$ for UT $i$ \\
\hline$P_{m, i}^{D}$ & Transmission power of BS $m$ for UT $i$ \\
\hline$B_{m}^{D}$ & Total bandwidth of BS $m$ for DL \\
\hline$B_{m}^{U}$ & Total bandwidth of BS $m$ for UL \\
\hline$P_{m}^{D}$ & Total transmission power of BS $m$ \\
\hline $\mathrm{PL}\left(d_{m, i}\right)$ & Pathloss between BS $m$ and UT $i$ \\
\hline$P_{i}^{U}$ & Transmission power of UT $i$ \\
\hline$P_{\max }^{U}$ & Maximum transmission power of a UT \\
\hline
\end{tabular}

where $I_{i}^{D}$ is the interference at the UT $i, N_{f}$ is the receiver noise figure and $N_{0}$ is the noise power spectral density. The pathloss term $\operatorname{PL}\left(d_{m, i}\right)$ contains large scale statistics as pathloss and log-normal shadowing. It can be expressed like $\operatorname{PL}\left(d_{m, i}\right)=c+10 n \log \left(d_{m, i}\right)+X_{\sigma}$ where $c$ is the power loss at $1 \mathrm{~m}$ away from the transmitter, $n$ is the pathloss coefficient and $X_{\sigma}$ is added due to shadowing effect which is a zero-mean Gaussian distributed random variable with standard deviation $\sigma$ in $\mathrm{dB}$. The definition of the interference in the formulation does not reflect the instantaneous interference, because the consideration of the instantaneous interference cannot be thought apart from interference management and scheduling, which are out of the scope of this paper. We assume that the assignment of the bandwidth for each UT is done randomly without interference management and scheduling. In other words, each UT can cause interference for every portion of the bandwidth with some probability in a certain time. This ran- 
domness of the assignment can be included in the calculations by taking the average interference for the sake of simplicity. As a matter of fact, our assumption corresponds to the worse case scenario because we can decrease interference by the help of interference management and scheduling. In that respect, the average interference can be defined as $I_{i}^{D}=\sum_{\substack{k \in A \\ k \neq m}} \frac{P_{k}^{D}}{\mathrm{PL}\left(d_{k, i}\right)} \frac{B_{m, i}^{D}}{B_{B S}}$ where $i \in S_{m}$. The definition of the interference term for the uplink case is similar to the downlink case, namely we can use the average interference for a UT instead of instantaneous interference. So, we can define the interference at BS $m$ for UT $i$ in the uplink transmission as $I_{i}^{U}=\sum_{\substack{j=1 \\ j \notin S_{m}}}^{N} \frac{P_{j}^{U}}{\operatorname{PL}\left(d_{m, j}\right)} \frac{B_{m, i}^{U}}{B_{B S}}$. As a result the uplink data rate of a UT is given by

$$
R_{u l}=B_{m, i}^{U} \log \left(1+\frac{P_{i}^{U} / \mathrm{PL}\left(d_{m, i}\right)}{I_{i}^{U}+N_{0} N_{f} B_{m, i}^{U}}\right) .
$$

\section{A. Power Control}

In a network, the optimum power control should be done according to SINR levels of all UTs. However, to determine the exact interference and to solve the power allocation problem for all users accordingly in a single shot is very hard, if possible. Instead, we propose a suboptimum power control method which works iteratively. Our method needs to know the power allocation for a single cell where the interference is assumed constant. So the optimization problem for the cell $m$ is given by

$$
\begin{array}{ll}
\operatorname{minimize} & P_{m}^{D} \\
\text { subject to }: & B_{m}^{D} \leq B_{B S} .
\end{array}
$$

where $P_{m}^{D}=\sum_{i \in S_{m}} P_{m, i}^{D}$ and $B_{m}^{D}=\sum_{i \in S_{m}} B_{m, i}^{D}$. In fact, there should be one more constraint in the optimization problem formulation which is $P_{m}^{D} \leq P_{B S}$. However, in some occasions, all the UTs of a BS cannot be served by the limited power of the BS. In that case, the worst UT in terms of received signal power is blocked and the power allocation is refound for non-blocked UTs. So, we exclude this constraint and check the total power of the BS after the power allocation. Then, if necessary, we block some of the UTs one by one.

We can find the solution of the optimization problem by the help of Lagrangian method. The Lagrangian of (4) for a given BS $m$ can be written as

$$
\begin{aligned}
L= & \sum_{i \in S_{m}}\left(2^{R_{d l} / B_{m, i}^{D}}-1\right) \gamma_{i} \operatorname{PL}\left(d_{m, i}\right) B_{m, i}^{D} \\
& +\lambda\left(\sum_{i \in S_{m}} B_{m, i}^{D}-B_{B S}\right)
\end{aligned}
$$

where $\lambda$ is the Lagrange multiplier and $\gamma_{i}=$ $\sum_{\substack{k \in A \\ k \neq m}} \frac{P_{k}^{D}}{B_{B S} \mathrm{PL}\left(d_{k, i}\right)}+N_{0} N_{f}$. We put $P_{m}^{D}$ in terms of $B_{m, i}^{\substack{k \neq m \\ D}}$ by using (2) because we need to take the derivative of the Lagrangian with respect to $B_{m, i}^{D}$. When we take the derivative of the Lagrangian and equalize it to zero, we get

$$
\lambda=\gamma_{i} \operatorname{PL}\left(d_{m, i}\right)\left(1+e^{R_{d l} / B_{m, i}^{D}}\left(\frac{R_{d l} \ln 2}{B_{m, i}^{D}}-1\right)\right),
$$

where that is valid for $\forall i \in S_{m}$. According to KKT conditions $\lambda\left(\sum_{i \in S_{m}} B_{m, i}^{D}-B_{B S}\right)=0$ and when $\lambda$ is nonzero, $\sum_{i \in S_{m}} B_{m, i}^{D}=B_{B S}$. Since (4) is a non-convex problem, the analysis of the KKT system gives a suboptimum power allocation. We consider LTE networks where only discrete bandwidth allocation is possible. In LTE standards a resource block is $180 \mathrm{kHz}$ and consists of 12 subchannels. A subchannel, which is $15 \mathrm{kHz}$, should be allocated to a single UT. So we have a discrete search space and the Algorithm 1 quickly finds the suboptimum power allocation. The same procedure is also valid for uplink transmission. We skip the calculations of suboptimum power allocation for uplink transmission, because it is very similar to the downlink case. The given pseudocode given in Algorithm 1 is just for a single cell. We can find the power allocation for all the cells by using this algorithm iteratively. We fix the interference and find the power allocation for every cell at each iteration, then update the interference and repeat the same process until allocated power converge.

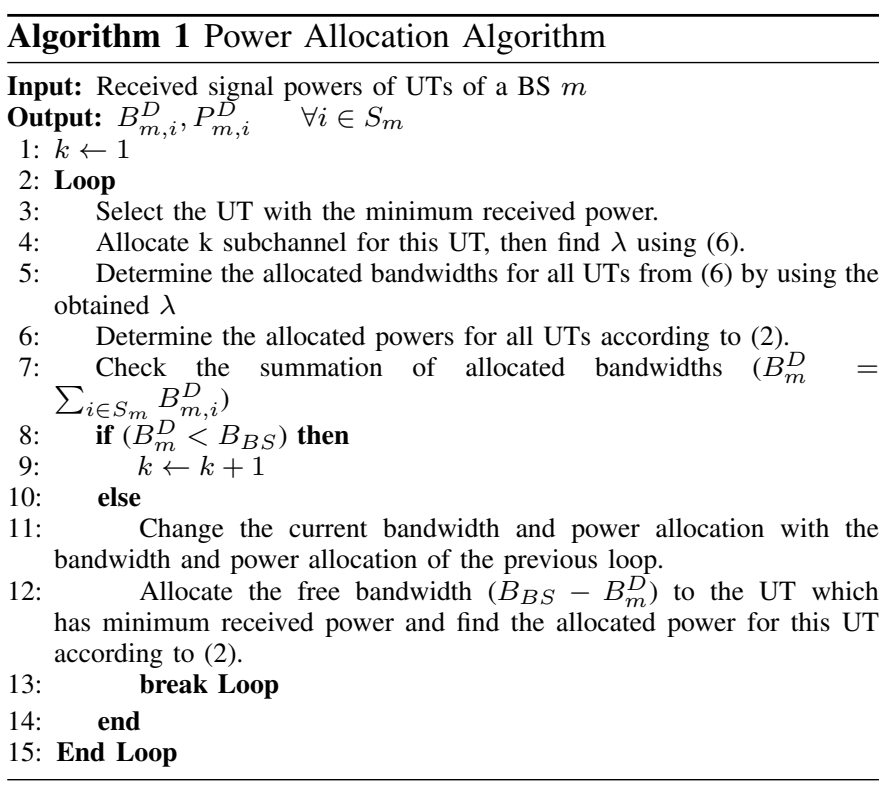

\section{B. Heuristic Algorithm}

We want to switch off as many BSs as possible without violating the QoS constraint. At the same time, the proposed algorithm should be aware of UTs' power consumption. There is a trade off between the total power consumption of UTs and the total power consumption of the network. It cannot be solved in polynomial time to check all the possible network configurations to find the optimum one. Therefore, we propose a heuristic algorithm, we call as user-aware CSO algorithm, which tries to switch off BSs one by one like the cell-zooming algorithm does. The cell-zooming algorithm sorts the BSs 
according to their traffic loads and switches off BSs starting from the one that has the least traffic load. Mainly, the sorting criterion is different in our proposed algorithm. How much the sum-power of UTs increases when a BS is switched off is used as sorting criterion.

When one of the BS is switched off, its UTs are served by neighbor BSs and each UT of switched off BS attaches to a neighbour BS with highest received power. While the pathloss between the neighbor BS and the UT is higher than original BS and the UT, the transmission power of the UT should increase. We can denote the increment of the sumpower of UTs as $\Delta P_{S, m}=P_{S}-P_{S, m}$ where $P_{S}=\sum_{i}^{N} P_{i}^{U}$ is the current sum-power of UTs and $P_{S, m}$ is the sum-power of UTs when BS $m$ is switched off. We propose a heuristic algorithm where the sorting criteria is the increment of the sum power of the UTs when one of the BSs is switched off. So we can switch off BSs starting from which has the least $\Delta P_{S, m}$ instead of which has the least traffic load. We call these algorithms as "user-aware CSO algorithm".

The proposed CSO process can be performed by the help of some additional signalling. For example, we assume that the locations of BSs and UTs are known by the central station. Therefore, in some periods of time, the information about network conditions, i.e., locations of active BSs and UTs, is collected by the central station. Then, the BSs that should be switched off are determined according to the proposed heuristic algorithm and all the BSs are informed about which BSs are going to be switched off. Finally, the necessary handover process is completed before the CSO process is performed.

We need some new variables to build the user-aware CSO algorithm:

- $\mathbf{X}=\left[x_{m, i}\right]_{N \times N_{A}}$ where $x_{m, i} \in\{0,1\} . x_{m, i}$ is a variable that takes 1 when BS $m$ is associated with UT $i$ and takes 0 otherwise.

- $\mathbf{W}=\left[w_{m, i}^{D}\right]_{N \times N_{A}}$ where $w_{m, i}^{D}$ is the received signal power of UT $i$ when the signal is sent from BS $m$.

- $\mathbf{B}^{\mathbf{D}}=\left[B_{m, i}^{D}\right]_{N \times N_{A}}$ shows the allocated bandwidths for DL.

- $\mathbf{B}^{\mathbf{U}}=\left[B_{m, i}^{U}\right]_{N \times N_{A}}$ shows the allocated bandwidths for UL.

- $\mathbf{P}^{\mathbf{D}}=\left[P_{m, i}^{D}\right]_{N \times N_{A}}$ shows the allocated powers of BSs for DL.

- $\mathbf{P}^{\mathbf{U}}=\left[P_{i}^{U}\right]_{N \times 1}$ shows the power levels of UTs.

- $\mathbf{I}^{\mathbf{D}}=\left[I_{i}^{D}\right]_{N \times 1}$ shows the interference for DL.

- $\mathbf{I}^{\mathbf{U}}=\left[I_{i}^{U}\right]_{N \times 1}$ shows the interference for UL.

- $\mathbf{P}_{\text {dif }}=\left[\Delta P_{S, m}\right]_{1 \times N_{A}}$ where $\Delta P_{S, m}$ shows the sumpower change of whole UTs when BS $m$ is switched off.

The pseudo-code of the proposed user-aware CSO algorithm is given in Algorithm 2.

\section{Performance Results}

The system parameters are listed in Table II. These parameter values are taken from [15] and [8] which are consistent with LTE networks.

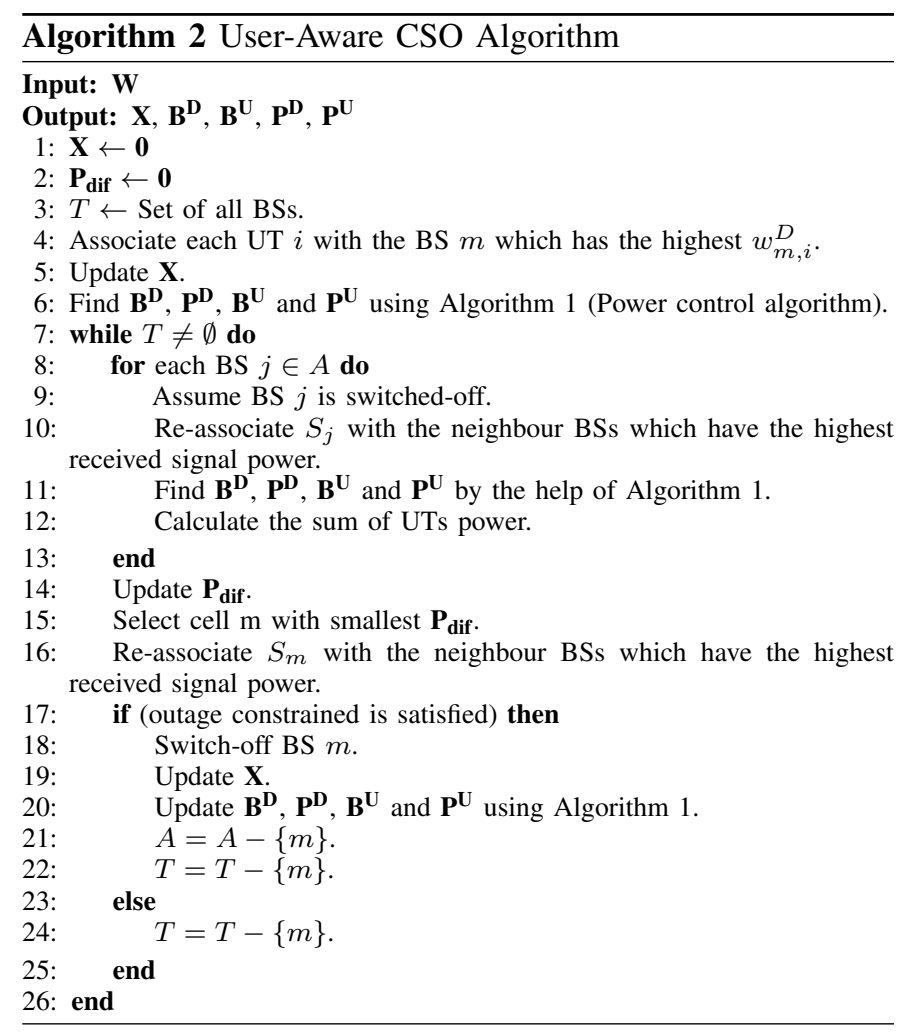

TABLE II: System parameters

\begin{tabular}{|l|l|}
\hline Total bandwidth of a BS $\left(B_{B S}\right)$ & $5 \mathrm{MHz}$ \\
\hline BS maximum transmission power $\left(P_{B S}\right)$ & $5 \mathrm{~W}$ \\
\hline UT max. transmission power $\left(P_{\max }^{U}\right)$ & $250 \mathrm{~mW}$ \\
\hline Path loss model & $30+36.7 \log (d)+X_{\sigma}$ \\
\hline Standard deviation of $X_{\sigma}(\sigma)$ & $8 \mathrm{~dB}$ \\
\hline UT downlink data rate $\left(R_{d l}\right)$ & $500 \mathrm{kbps}$ \\
\hline UT uplink data rate $\left(R_{u l}\right)$ & $300 \mathrm{kbps}$ \\
\hline Thermal noise $\left(N_{0}\right)$ & $-174 \mathrm{dBm} / \mathrm{Hz}$ \\
\hline Noise figure $\left(N_{f}\right)$ & $10 \mathrm{~dB}$ \\
\hline Inter BS distance & $500 \mathrm{~m}$ \\
\hline Min. distance between a UT and a BS & $10 \mathrm{~m}$ \\
\hline Number of BSs $(M)$ & 25 \\
\hline Number of UTs & $50,125,200$ \\
\hline Max. outage probability (total) & $2 \%$ \\
\hline$\alpha$ and $\beta$ in $(1)$ & $3.1 \mathrm{~W}$ and $53 \mathrm{~W}$ \\
\hline
\end{tabular}

Fig. 2 represents the sum power of UTs versus the number of switched off BSs for 2 algorithms: user-aware CSO algorithm and improved cell-zooming algorithm. The improved cell-zooming algorithm is used as a reference case in the simulations. Fig. 2 shows the simulation results for different total number of users as 50,125 and 200. The proposed algorithm has roughly $43 \%, 40 \%$ and $38 \%$ less sum power of UTs than improved cell-zooming algorithm when the number of users is 50,125 and 200, respectively. When we compare the algorithms in terms of switching-off capabilities, we observe from Fig. 3 that the user-aware CSO algorithm is slightly better than the improved cell-zooming algorithm. Namely, the 


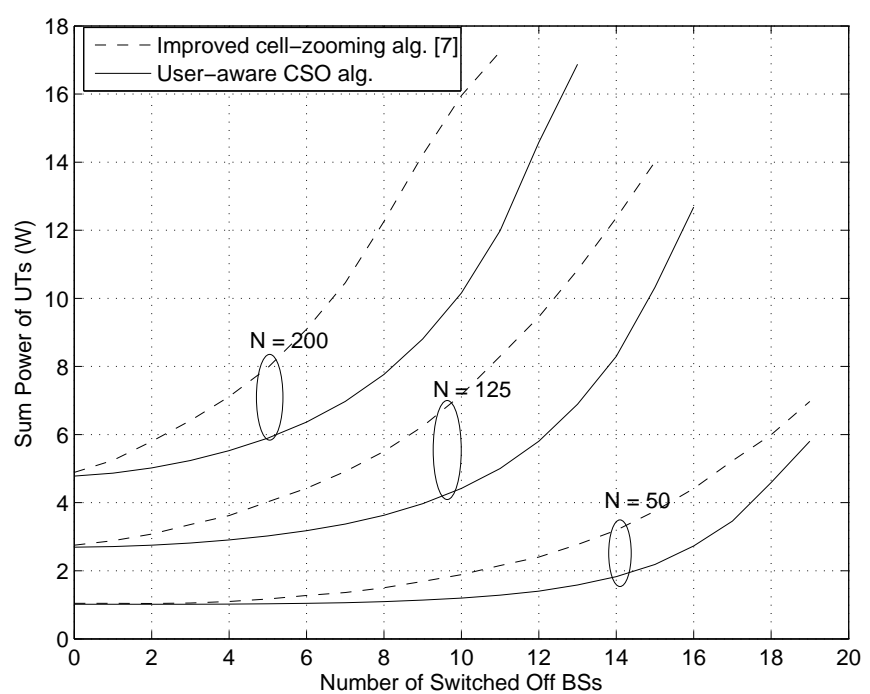

Fig. 2: The number of switched off BSs vs. the sum power of UTs for the considered algorithms where total number of users $(N)$ is 50,125 and 200.

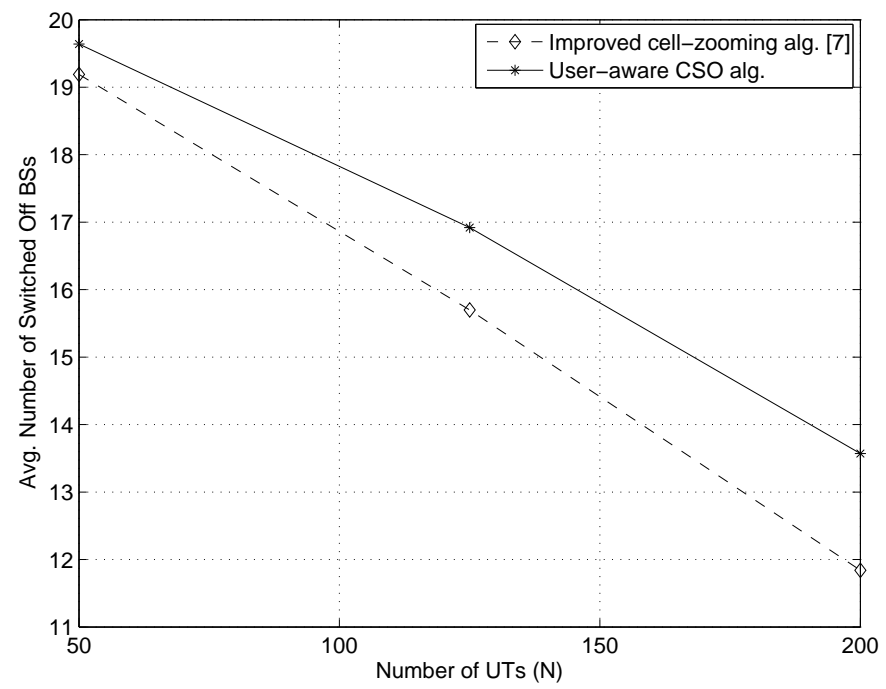

Fig. 3: CSO capabilities of the considered algorithms with respect to the number of UTs in the network $(N)$.

proposed algorithm can switch off 0.5-1.8 more BSs than the reference algorithm in average where the number of users is between 50 and 200 .

Another important point about the simulation results is that the results are completely dependent on the system parameters given in Table II. For example the total bandwidth of the BS, downlink and uplink rate requirements can be chosen differently and that gives very different results. Fig. 4 and Fig. 5 represent the reaction of considered algorithms to the change of the uplink data rate requirement. It is observed that the behaviour of the CSO capabilities of both algorithms are very similar under uplink data rate requirement change. The number

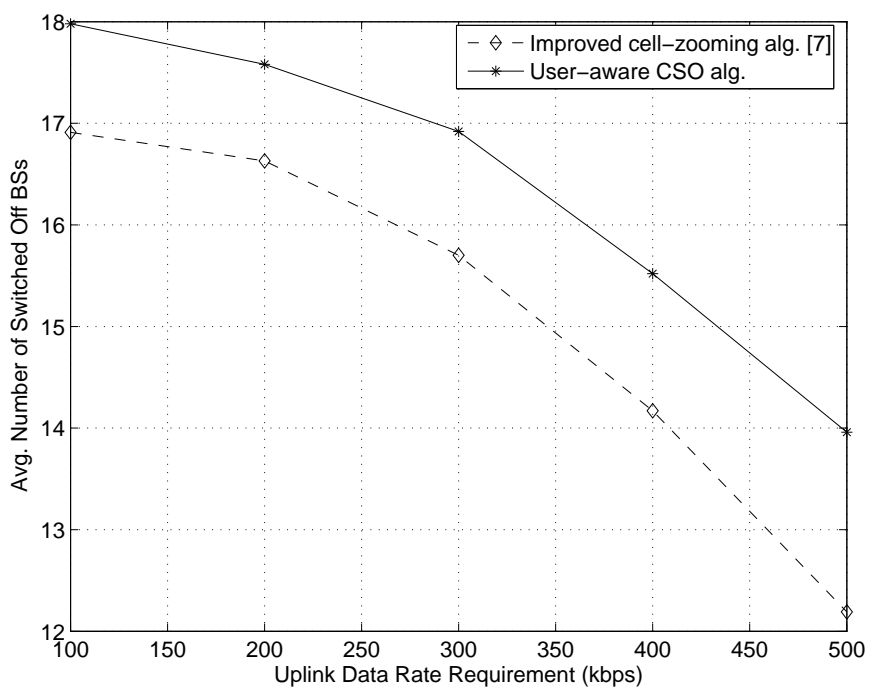

Fig. 4: CSO capabilities of the considered algorithms with respect to different uplink data rate requirement values.

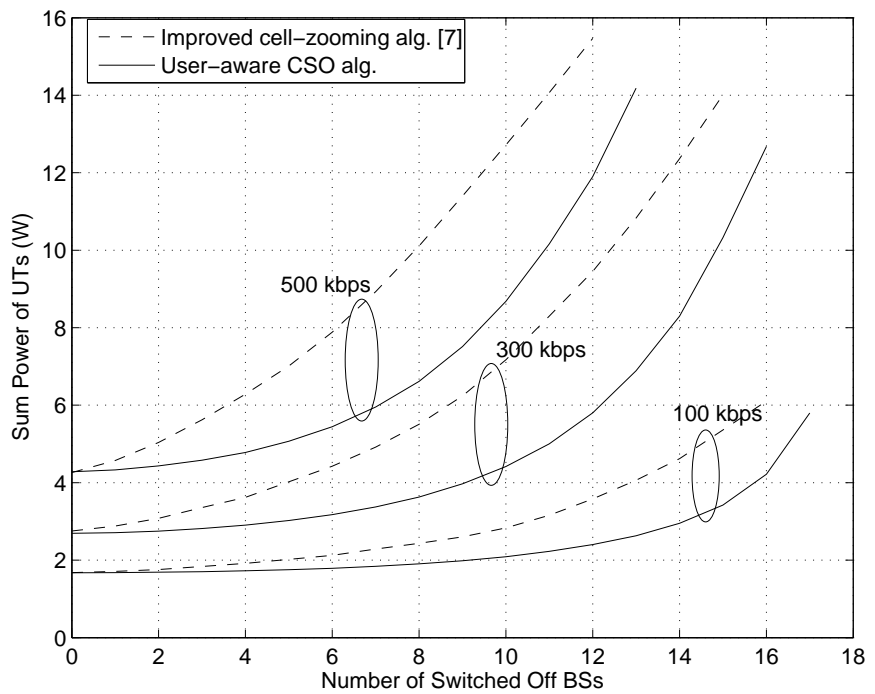

Fig. 5: Sum power of UTs for the considered algorithms with respect to different uplink data rate requirement values.

of switched off BSs falls from 18 to 14 and from 16.9 to 12.3 gradually for the user-aware CSO algorithm and the improved cell-zooming algorithm, respectively, when the uplink data rate requirement increases to $500 \mathrm{kbps}$ from $100 \mathrm{kbps}$ as shown in Fig. 4. Fig. 5 represents that the proposed algorithm achieves up to between $35 \%$ and $40 \%$ less sum power of UTs than the reference algorithm for different uplink data rate requirements.

\section{Conclusions And COMments}

Recently, energy efficiency has become an important concern in cellular networks. The number of BSs will continue to increase in the upcoming $5 \mathrm{G}$ networks for better connectivity and service; this will cause an increased power consumption. 
Therefore, there is a growing interest in CSO techniques which aim at minimizing the energy consumption. However, the effect of CSO techniques on the UT side has not been studied in the literature. We proposed a user-aware CSO algorithm where the power consumption of UTs are minimally impacted by switching off BSs. It is observed that the user-aware CSO algorithm achieves up to around $40 \%$ less power consumption of UTs in comparison to the improved cell-zooming algorithm given in [7].

\section{ACKNOWLEDGMENT}

The authors would like to thank Dr. Gamini Senarath and Dr. Ngoc Dao from Huawei Technologies Canada Co., Ltd. and Dr. Ramy H. Gohary from Carleton University for their valuable comments.

\section{REFERENCES}

[1] N. Chilamkurti, S. Zeadally, and F. Mentiplay, "Green networking for major components of information communication technology systems," EURASIP Journal on Wireless Communications and Networking, vol. 2009, article no. 35, pp. 1-7, Jan. 2009.

[2] M. Ajmone Marsan, L. Chiaraviglio, D. Ciullo, and M. Meo, "On the effectiveness of single and multiple base station sleep modes in cellular networks," Elsevier Computer Networks, vol. 57, pp. 3276-3290, July 2013.

[3] I. Humar, X. Ge, L. Xiang, M. Jo, M. Chen, and J. Zhang, "Rethinking energy efficiency models of cellular networks with embodied energy," IEEE Network, vol. 25, no. 2, pp. 40-49, Mar. 2011.

[4] K. Son and B. Krishnamachari, "SpeedBalance: speed-scaling-aware optimal load balancing for green cellular networks," in IEEE INFOCOM, Mar. 2012.

[5] U. Paul, A.P. Subramanian, M.M. Buddhikot, and S.R. Das, "Understanding traffic dynamics in cellular data networks," in IEEE INFOCOM, April 2011, pp. 882-890.

[6] Z. Niu, Y. Wu, J. Gong, and Z. Yang, "Cell zooming for cost-efficient green cellular networks," IEEE Comm. Mag., vol. 48, no. 11, pp. 74-79, Nov. 2010.

[7] F. Alaca, A. Bin Sediq, and H. Yanikomeroglu, "A genetic algorithm based cell switch-off scheme for energy saving in dense cell deployments," in IEEE Globecom Workshops, Dec. 2012.

[8] A. Bousia, A. Antonopoulos, L. Alonso, and Ch. Verikoukis, " "Green" distance-aware base station sleeping algorithm in LTE-Advanced ", in IEEE ICC, June 2012.

[9] T. Kang, X. Sun, and T. Zhang, "Base station switching based dynamic energy saving algorithm for cellular networks," in 3rd IEEE International Conference on Network Infrastructure and Digital Content (IC-NIDC 2012), Sep. 2012.

[10] Y. Zhu, T. Kang, T Zhang, and Z. Zeng, "QoS-aware user association based on cell zooming for energy efficiency in cellular networks," in IEEE Inter. Symp. Personal, Indoor and Mobile Radio Commun. (PIMRC), Sep. 2013.

[11] C. Turyagyenda, K. Al-Begain, and N. Albeiruti, "A novel sleep mode operation for energy efficient LTE cellular networks: A sum product algorithm implementation," in 7th International Conference on Next Generation Mobile Apps, Services and Technologies (NGMAST), Sep. 2013.

[12] M. Ajmone Marsan, L. Chiaraviglio, D. Ciullo, and M. Meo, "Optimal energy savings in cellular access networks," in IEEE ICC, June 2009.

[13] Bousia, E. Kartsakli, L. Alonso, and C. Verikoukis, "Energy efficient base station maximization switch off scheme for LTE-advanced," in 17th IEEE International Workshop on Computer Aided Modeling and Design of Communication Links and Networks (CAMAD), Sep. 2012.

[14] D. Gonzalez G, H. Yanikomeroglu, M. Garcia-Lozano, and S. R. Boque, "A novel multiobjective framework for cell switch-off in dense networks", in IEEE ICC, June 2014.

[15] S. Morosi, P. Piunti, and E. Del Re, "Improving cellular network energy efficiency by joint management of sleep mode and transmission power," in 24th Tyrrhenian International Workshop on Digital Communications (TIWDC) on "Green ICT", Sep. 2013. 